T. Sugatani

Nagoya Math. J.

Vol. 81 (1981), 73-78

\title{
RINGS OF CONVERGENT POWER SERIES AND WEIERSTRASS PREPARATION THEOREM
}

\author{
TAKASI SUGATANI
}

§.

Let $B$ be a $B$-ring with a nonarchimedean valuation | |, i.e., $B$ is an integral domain satisfying the following conditions: (i) $B$ is bounded $(|a| \leqq 1$ for every $a \in B$ ), (ii) the boundary $\partial(B)=\{a \in B ;|a|=1\}$ forms a multiplicative group. Let $Z_{+}$denote the set of all nonnegative integers. Let $n \in Z_{+}$. Let $x_{1}, \cdots, x_{n}$ be $n$ variables over $B$. We denote by $A_{n}=$ $B\left\langle x_{1}, \cdots, x_{n}\right\rangle$ the set of all elements which can be written in the form

$$
\sum_{\nu} a_{\nu} x^{\nu}
$$

where $a_{\nu} \in B$ for all $\nu \in Z_{+}^{n}$ and $\left|a_{\nu}\right| \rightarrow 0$ as $\nu_{1}+\cdots+\nu_{n} \rightarrow \infty$. We define a norm \|\| on $A_{n}$ : For $g=\sum a_{\nu} x^{\nu} \in A_{n}$, let $\|g\|=\max \left\{\left|a_{\nu}\right|\right\}$. Let $m$ be the maximal ideal of $B$ and $k=B / m$ be the residue field. Let $\tau$ be the canonical mapping of $B$ onto $k$. Then $\tau$ can be extended to an epimorphism from $A_{n}$ to a polynomial ring $k\left[x_{1}, \cdots, x_{n}\right]$ in the usual manner. We assume, throughout this paper, the $B$-ring $B$ is complete. We shall identify $A_{n-1}\left\langle x_{n}\right\rangle$ with $A_{n}$ so that each element $g$ of $A_{n}$ has an expression $\sum g_{i} x_{n}^{i}$, where $g_{i} \in$ $A_{n-1}$ for all $i \in Z_{+}$and $\left\|g_{i}\right\| \rightarrow 0$ as $i \rightarrow \infty$. For any $s \in Z_{+}$, let $P_{s}$ denote the set of all polynomials of $A_{n-1}\left[X_{n}\right]$ of degree $<s$. One can see several properties on a $B$-ring in [2], [4].

In this paper, we shall prove Weierstrass Preparation Theorem for $A_{n}$. We shall obtain Weierstrass Form Theorem and Scherung Theorem for $A_{n}$ also.

Before going into discussions, the author wishes to express his thanks to Professor Hisasi Morikawa for his valuable suggestions and constant encouragement in the presentation of this paper.

Received June 1, 1979. 
$\S 1$.

To consider Weierstrass Preparation Theorem we need some information on unit elements of $A_{n}$. We prove

Proposition 1.1. Let $g=\sum a_{\nu} x^{\nu} \in A_{n}$. Then $g$ is a unit element of $A_{n}$ if and only if

$$
\left\{\begin{array}{l}
\left|a_{0, \cdots, 0}\right|=1 \\
\left|a_{\nu}\right|<1 \text { for each } \nu \neq(0, \cdots, 0) .
\end{array}\right.
$$

Proof. Let $g$ be a unit element of $A_{n}$ then there exists an element $u$ of $A_{n}$ such that $g u=1$. It follows

$$
1=\tau(g u)=\tau(g) \tau(u) \in k\left[x_{1}, \cdots, x_{n}\right] .
$$

Hence (1.1) holds. Conversely, suppose $g$ satisfies (1.1). Then it can be seen that the inverse element of $g$ is given by

$$
g^{-1}=\left(a_{0, \ldots, 0}\right)^{-1}\left[1+\sum_{1}^{\infty}\left(g^{\prime \prime}\right)^{i}\right],
$$

where

$$
-g^{\prime \prime}=\left(a_{0, \ldots, 0}\right)^{-1} \sum_{\nu \neq(0, \ldots .0)} a_{v} x^{\nu}
$$

With this the proof is complete.

From this proposition, we have the followings:

Remark 1.2. If $n \geqq 1$, then $A_{n}$ is not a quasi-local ring.

Proof. For a contradiction, we assume $A_{n}$ is a quasi-local ring. Then it follows the set $M$ of all nonunit elements of $A_{n}$ forms a maximal ideal. By Proposition 1.1, for instance, $x_{1}$ and $1+x_{1}$ belong to $M$. Then 1 belongs to $M$, a contradiction.

Let $g=\sum g_{i} x_{n}^{i} \in A_{n-1}\left\langle x_{n}\right\rangle$. Let $s \in Z_{+}$. We say that $g$ is general (allgemein) in $x_{n}$ of order $s$ if $g_{s}$ is a unit element of $A_{n-1}$ and $\left\|g_{i}\right\|<1$ for all $i>s$.

Remark 1.3. $g \in A_{n}$ is general in $x_{n}$ of order $s \geqq 0$ if and only if

$$
\tau(g)=\tau\left(g_{0}\right)+\tau\left(g_{1}\right) x_{n}+\cdots+\tau\left(g_{s}\right) x_{n}^{s}
$$

for which $\tau\left(g_{i}\right) \in k\left[x_{1}, \cdots, x_{n-1}\right]$ for each $i=0, \cdots, s-1$, and $\tau\left(g_{s}\right) \in k^{*}=$ $k-\{0\}$. 
Proof. By Proposition 1.1, it is clear $g_{s}$ is a unit element of $A_{n-1}$ if and only if $\tau\left(g_{s}\right)$ is in $k^{*}$. It is easy to verify the other conditions on the coefficients of $g$.

\section{$\S 2$.}

In this section we shall show Weierstrass Form for $A_{n}$, which is a generalization of the result of Grauert-Remmert [1].

Theorem 2.1 (Weierstrass Form for $A_{n}$ ). Let $g \in A_{n}$ be general in $x_{n}$ of order $s \geqq 0$. Then for each $f \in A_{n}$ there exists a unique pair $q \in A_{n}, r \in$ $P_{s}$ satisfying

$$
f=q g+r
$$

Further, we have

$$
\|f\|=\max \{\|q\|,\|r\|\} .
$$

In order to prove this theorem we need the following lemmas. Lemma 2.3 is established for $K\left\langle x_{1}, \cdots, x_{n}\right\rangle$ (Satz 2.1 of [1]). But in our case it cannot be assumed that for a nonzero element $f$ of $K\left\langle x_{1}, \cdots, x_{n}\right\rangle$ there exists a nonzero element $a$ in $K$ satisfying $\|a f\|=1$, because we take an arbitrary $B$-ring $B$ as a coefficient ring. So, we prove at first Lemma 2.2 analogous to Theorem 3.20 in [3].

Lemma 2.2. Let $g \in A_{n}$ be general in $x_{n}$ of order $s \geqq 0$. Then for $q \in$ $A_{n}$ and $r \in P_{s}$ we have

$$
\|q g+r\| \geqq\|q\| \text {. }
$$

Proof. Let $q=\sum b_{\nu} x^{\nu} \in A_{n}$. Let $\mu=\left(\mu_{1}, \cdots, \mu_{n}\right) \in Z_{+}^{n}$ be the heighest indexterm of $\nu$ such that $\|q\|=\left|b_{\nu}\right|$. If $q g=\sum c_{\nu} x^{\nu} \in A_{n}$ then $\|q\|=\|q g\|$ $=\left|c_{\mu^{\prime}}\right|$, where $\mu^{\prime}=\left(\mu_{1}, \cdots, \mu_{n-1}, \mu_{n}+s\right)$. If $r \in A_{n}$ such that the coefficient of $x^{\mu^{\prime}}$ vanishes, then $\|q g+r\| \geqq\|q\|$. In particular this is true for all $r \in$ $P_{s}$ and now (2.3) follows.

LEMMA 2.3. Let $g \in A_{n-1}\left[x_{n}\right]$ be of degree $s$ and the leading coefficient be a unit element of $A_{n-1}$. Then for each $f \in A_{n}$ there exists a pair $q \in A_{n}$, $r \in P_{s}$ satisfying

$$
f=q g+r .
$$

Proof. Let $f=\sum f_{i} x_{n}^{i} \in A_{n-1}\left\langle x_{n}\right\rangle$. It follows for each $i \geqq 0$ there exist 
$q_{i}$ and $r_{i} \in A_{n-1}\left[x_{n}\right]$ such that $r_{i}$ is of degree $<s$ and $f_{i} x_{n}^{i}=q_{i} g+r_{i}$. Then (2.3) implies

$$
\left\|f_{i}\right\|=\max \left\{\left\|q_{i}\right\|,\left\|r_{i}\right\|\right\} .
$$

Let $r=\sum_{0}^{\infty} r_{i}$ and $q=\sum_{0}^{\infty} q_{i}$. Then we see that $q \in A_{n}$ and $r \in P_{s}$. With these $q$ and $r$ we obtain the equation (2.4).

Proof of Theorem 2.1. If $r \in g A_{n} \cap P_{s}$ then by Lemma 2.2, $0=\|r-r\|$ $\geqq\|r\|$. Therefore we have

$$
g A_{n} \cap P_{s}=0,
$$

which shows the uniqueness of a pair $q, r$ of (2.1).

We next prove

$$
A_{n}=g A_{n}+P_{s},
$$

by using Grauert-Remmert's method in [1]. In fact, let $g=\sum g_{i} x_{n}^{i} \in$ $A_{n-1}\left\langle x_{n}\right\rangle$ and let $g=g^{(1)}+g^{(2)}$, where $g^{(1)}=\sum_{0}^{s} g_{i} x_{n}^{i}$. Then we have $\delta=$ $\left\|g^{(2)}\right\|<1$. We define a set of elements $f_{j}, q_{j}$ and $r_{j}$ of $A_{n}$ in the following way: Let $f_{0}=f=q_{0} g^{(1)}+r_{0}$, where $r_{0} \in P_{s}$. For $j \in Z_{+}$we put $f_{j+1}=f_{j}-$ $q_{j} g-r_{j}=q_{j+1} g^{(1)}+r_{j+1}$, where $r_{j+1} \in P_{s}$. This procedure is possible by Lemma 2.3. Then it follows

$$
f_{j+1}=-q_{j} g^{(2)}
$$

whence, by (2.5)

$$
\left\|f_{j+1}\right\|=\delta\left\|q_{j}\right\| \leqq \delta\left\|f_{j}\right\|
$$

therefore we have

$$
\left\|f_{j+1}\right\| \leqq \delta\left\|f_{j}\right\|
$$

By induction on $j \geqq 0$, we have

$$
\left\|f_{j}\right\| \leqq \delta^{j}\|f\|,\left\|q_{j}\right\| \leqq \delta^{j}\|f\|
$$

and

$$
\left\|r_{j}\right\| \leqq \delta^{j}\|f\|
$$

Putting $q=\sum_{0}^{\infty} q_{j}$ and $r=\sum_{0}^{\infty} r_{j}$, we have $q \in A_{n}$ and $r \in P_{s}$ satisfying (2.7) as required.

By the definition it is clear $\|q\| \leqq\|f\|$. Then we see $\|r\|=\|f-q g\| \leqq$ $\max \{\|f\|,\|q\|\}=\|f\|$. Therefore we have $\|f\| \geqq \max \{\|q\|,\|r\|\}$. This proves 
half of (2.2) and the other half is obvious. Thus our theorem is completely proved.

$\S 3$.

Theorem 3.1 (Weierstrass Preparation Theorem for $A_{n}$ ). Let $g \in A_{n}$ be general in $x_{n}$ of order $s \geqq 0$. Then there exist uniquely $u, a_{0}, \cdots, a_{s-2}$ and $a_{s-1}$ satisfying the following conditions: $u$ is a unit element of $A_{n}, a_{0}, \cdots, a_{s-1}$ are in $A_{n-1}$ and

$$
g=u\left(x_{n}^{s}+a_{s-1} x_{n}^{s-1}+\cdots+a_{1} x_{n}+a_{0}\right) .
$$

Proof. By Theorem 2.1 there exists a unique pair $q \in A_{n}, r \in P_{s}$ satisfying

$$
x_{n}^{s}=q g+r \text {. }
$$

Applying Theorem 2.1 again, this time with $x_{n}^{s}-r$ instead of g, we obtain a unique pair $q^{\prime} \in A_{n}, r^{\prime} \in P_{s}$ satisfying

$$
g=q^{\prime}\left(x_{n}^{s}-r\right)+r^{\prime} .
$$

Then

$$
g=q^{\prime} q g+r^{\prime}
$$

therefore we must have $q^{\prime} q=1$ and $r^{\prime}=0$. In particular we have

$$
g=q^{\prime}\left(x_{n}^{s}-r\right) .
$$

Put $-a_{0},-a_{1}, \cdots,-a_{s-1}$ as the coefficients of $r$ and $u=q^{\prime}$. The uniqueness follows from the choice of $r$, which shows our assertion.

$\S 4$.

In this section we prove Scherung Theorem for $A_{n}$.

THEOREM 4.1. Suppose the residue field $k$ of $B$ is infinite. Let $f$ be in $A_{n}$ and $\|f\|=1$. Then there exists a B-automorphism $\sigma$ of $A_{n}$ such that $\sigma(f)$ is general in $x_{n}$.

Proof. Let $f=\sum_{0}^{\infty} f_{j}$, where each $f_{j}$ is the $j$-th homogeneous part of f. Then there exists $f_{s}$ such that $\left\|f_{s}\right\|=1$ and $\left\|f_{j}\right\|<1$ for all $j>s$. Let $\tau\left(f_{s}\right)=\bar{f}_{s}$. Then $\bar{f}_{s}$ is a nonzero element of $k\left[x_{1}, \cdots, x_{n}\right]$. If $n=1$ then the assertion is clear. Assume $n \geqq 2$. By our assumption that $k$ is infinite, we can choose an element $\left(\bar{a}_{1}, \cdots, \bar{a}_{n-1}, \bar{a}_{n}\right) \in k^{n}$ satisfying 


$$
f_{s}\left(\bar{a}_{1}, \cdots, \bar{a}_{n}\right) \in k^{*},
$$

where $\bar{a}_{j}=\tau\left(a_{j}\right)$ for $a_{j} \in B, j=1, \cdots, n$. Here we may assume $\left|a_{n}\right|=1$. Put $b=f_{s}\left(a_{1}, \cdots, a_{n}\right)$. Then $|b|=1$. We define a $B$-algebra endomorphism $\sigma$ such as

$$
\begin{aligned}
& \sigma\left(x_{j}\right)=x_{j}+a_{j} x_{n}, \quad j=1, \cdots, n-1, \\
& \sigma\left(x_{n}\right)=x_{n} .
\end{aligned}
$$

Then $\sigma^{-1}$ is given by

$$
\begin{aligned}
& \sigma^{-1}\left(x_{j}\right)=x_{j}-a_{\jmath} x_{n}, \quad j=1, \cdots, n-1, \\
& \sigma^{-1}\left(x_{n}\right)=x_{n} .
\end{aligned}
$$

It can be seen by easy calculations

$$
\sigma(f)=\sum_{0}^{\infty} f_{i}^{*} x_{n}^{i},
$$

where each $f_{i}^{*} \in A_{n-1}$ and $\left\|f_{i}^{*}\right\|<1$ for all $i>s$. In particular $f_{s}^{*}$ is a unit element of $A_{n-1}$, for the constant term is equal to $b$ and the norm of the part of terms of degree $\geqq 1$ is less than 1 . Therefore $\sigma(f)$ is general in $x_{n}$ of order $s$. Thus $\sigma$ is the $B$-automorphism to be desired.

\section{REFERENCES}

[1] H. Grauert and R. Remmert, Nichtarchimedische Funktionentheorie, WeierstrassFestschrift, Wissenschaftl. Abh. Arbeitsgem. f. Forsch. Nordrhein-Westfalen, 33 (1966), 393-476.

[2] H. Grauert and R. Remmert, Über die Methode der diskret bewerteten Ringe in der nichtarchimedischen Analysis, Inventiones Math., 2 (1966), 87-133.

[ 3 ] A. C. M. van Rooij, Non-Archimedean Functional Analysis, Marcel Dekker, Ine., New York, 1978.

[4] T. Sugatani, On quasi-noetherian rings which are not noetherian, Math. Rep. Toyama Univ., 1 (1978), 65-73.

Department of Mathematics

Faculty of Science

Toyama University 